\title{
Formalization of the Arithmetization of Euclidean Plane Geometry and Applications
}

\author{
Pierre Boutry, Gabriel Braun, Julien Narboux \\ ICube, UMR 7357 CNRS, University of Strasbourg \\ Pôle API, Bd Sébastien Brant, BP 10413, 67412 Illkirch, France
}

\begin{abstract}
This paper describes the formalization of the arithmetization of Euclidean plane geometry in the Coq proof assistant. As a basis for this work, Tarski's system of geometry was chosen for its well-known metamathematical properties. This work completes our formalization of the two-dimensional results contained in part one of the book by Schwabhäuser Szmielew and Tarski Metamathematische Methoden in der Geometrie. We defined the arithmetic operations geometrically and proved that they verify the properties of an ordered field. Then, we introduced Cartesian coordinates, and provided characterizations of the main geometric predicates. In order to prove the characterization of the segment congruence relation, we provided a synthetic formal proof of two crucial theorems in geometry, namely the intercept and Pythagoras' theorems. To obtain the characterizations of the geometric predicates, we adopted an original approach based on bootstrapping: we used an algebraic prover to obtain new characterizations of the predicates based on already proven ones. The arithmetization of geometry paves the way for the use of algebraic automated deduction methods in synthetic geometry. Indeed, without a "back-translation" from algebra to geometry, algebraic methods only prove theorems about polynomials and not geometric statements. However, thanks to the arithmetization of geometry, the proven statements correspond to theorems of any model of Tarski's Euclidean geometry axioms. To illustrate the concrete use of this formalization, we derived from Tarski's system of geometry a formal proof of the nine-point circle theorem using the Gröbner basis method. Moreover, we solve a challenge proposed by Beeson: we prove that, given two points, an equilateral triangle based on these two points can be constructed in Euclidean Hilbert planes. Finally, we derive the axioms for another automated deduction method: the area method.
\end{abstract}

Key words: Formalization, geometry, Coq, arithmetization, intercept theorem, Pythagoras' theorem, area method

\footnotetext{
* Extended version of a paper presented at SCSS 2016. Section 3 contains new material.

Email addresses: boutry@unistra.fr (Pierre Boutry), gabriel.braun@unistra.fr (Gabriel Braun), narboux@unistra.fr (Julien Narboux).
} 


\section{Introduction}

There are several ways to define the foundations of geometry. In the synthetic approach, the axiom system is based on some geometric objects and axioms about them. The best-known modern axiomatic systems based on this approach are those of Hilbert [Hil60] and Tarski [SST83]. In the analytic approach, a field $\mathbb{F}$ is assumed (usually $\mathbb{R}$ ) and the space is defined as $\mathbb{F}^{n}$. In the mixed analytic/synthetic approaches, one assumes both the existence of a field and also some geometric axioms. For example, the axiomatic systems proposed by the School Mathematics Study Group for teaching geometry in high-school [Gro61] in north America in the 60s are based on Birkhoff's axiomatic system [Bir32]. In this axiom system, the existence of a field to measure distances and angles is assumed. This is called the metric approach. A modern development of geometry based on this approach can be found in the books of Millman or Moise [MP91, Moi90]. The metric approach is also used by Chou, Gao and Zhang for the definition of the area method [CGZ94] (a method for automated deduction in geometry). Analogous to Birkhoff's axiomatic system, here the field serves to measure ratios of signed distances and areas. The axioms and their formalization in Coq can be found in [JNQ12]. Finally, in the relatively modern approach for the foundations of geometry, a geometry is defined as a space of objects and a group of transformations acting on it (Erlangen program [Kle93b, Kle93a]).

Although these approaches seem very different, Descartes proved that the analytic approach can be derived from the synthetic approach. This is called arithmetization and coordinatization of geometry. In [Des25] he defined addition, multiplication and square roots geometrically.

Our formalization of geometry consists in a synthetic approach based on Tarski's system of geometry. Readers unfamiliar with this axiomatic system may refer to [TG99] which describes its axioms and their history. Szmielew and Schwabhäuser have produced a systematic development of geometry based on this system. It constitutes the first part of [SST83]. We have formalized the two-dimensional results of the 16 chapters corresponding to this first part in the Coq proof assistant.

In this paper, we report on the formalization of the last three chapters containing the final results: the arithmetization and coordinatization of Euclidean geometry. It represents the culminating result of both [Hil60] and [SST83]. This formalization enables us to put the theory proposed by Beeson in [Bee13] into practice in order to obtain automatic proofs based on geometric axioms using algebraic methods. The arithmetization of geometry is a crucial result because, first, it guarantees that the axiomatic system captures the Euclidean plane geometry, and second it allows to use algebraic methods which are very powerful.

As long as algebra and geometry traveled separate paths their advance was slow and their applications limited. But when these two sciences joined company, they drew from each other fresh vitality, and thenceforth marched on at a rapid pace toward perfection.

(Joseph-Louis Lagrange, Leçons élémentaires sur les mathématiques; quoted by Morris Kline, Mathematical Thought from Ancient to modern Times, p. 322)

A formalization of the arithmetization of geometry and characterization of geometric predicates is also motivated by the need to exchange geometric knowledge data with a well-defined semantics. Algebraic methods for automated deduction in geometry have been integrated in dynamic geometry systems for a long time [Jan06, YCG08]. Automatic theorem provers can now be used by non-expert user of dynamic geometry systems such as GeoGebra which is used heavily in classrooms $\left[\mathrm{BHJ}^{+} 15\right]$. But, the results of these provers needs to be interpreted to understand in which geometry and under which assumptions they are valid. Different geometric constructions for the same statement can lead to various computation times and non-degeneracy conditions. 
Moreover, as shown by Botana and Recio, even for simple theorems, the interpretation can be non-trivial [BR16]. Our formalization, by providing a formal link between the synthetic axioms and the algebraic equations, paves the way for storing standardized, structured, and rigorous geometric knowledge data based on an explicit axiom system [CW13].

As far as we know, our library is the first formalization of the arithmetization of Euclidean plane geometry. However the reverse connection, namely that the Euclidean plane is a model of this axiomatized geometry, has been mechanized by Marić, Petrović, Petrović and Janičić [MPPJ12]. In [MP15], Marić and Petrović formalized complex plane geometry in the Isabelle/HOL theorem prover. In doing so, they demonstrated the advantage of using an algebraic approach and the need for a connection with a synthetic approach. Some formalization of Hilbert's foundations of geometry have been proposed by Dehlinger, Dufourd and Schreck [DDS01] in the Coq proof assistant, and by Dixon, Meikle and Fleuriot [MF03] using Isabelle/HOL. Likewise, a few developments based on Tarski's system of geometry have been carried out. For example, Richter, Grabowski and Alama have ported some of our Coq proofs to Mizar [RGA14] (46 lemmas). Moreover, Beeson and Wos proved 200 theorems of the first twelve chapters of [SST83] with the Otter theorem prover [BW17]. Finally, Durdevic, Narboux and Janičić [SDNJ15] generated automatically some readable proofs in Tarski's system of geometry. None of these formalization efforts went up to Pappus' theorem nor to the arithmetization of geometry.

The formalization presented in this paper is based on the library GeoCoq which contains a formalization of the first part of [SST83] and some additional results. This includes the proof that Hilbert's axiomatic system (without continuity) is bi-interpretable with the corresponding Tarski's axioms [BN12, BBN16], some equivalences between different versions of the parallel postulate [BGNS17], some decidability properties of geometric predicates [BNSB14a] and the synthetic proof of some popular high-school geometry theorems. The proofs are mainly manual, but we used the tactics presented in [BNSB14b, BNS15].

The arithmetization of geometry will allow us to link our formalization to the existing formalization of algebraic methods in geometry. For instance, the Gröbner basis method has already been integrated into Coq by Grégoire, Pottier and Théry [GPT11]. Furthermore, the area method [CGZ94] for non-oriented Euclidean geometry has been formalized in Coq as a tactic [Nar04, JNQ12]. Geometric algebras are also available in Coq and can be used to automatically prove theorems in projective geometry [FT11]. Finally, the third author together with Génevaux and Schreck have previously studied the integration of Wu's method [GNS11].

We first describe the formalization of the arithmetization of Euclidean geometry (Section 2). Then we provide the characterization of the main geometric predicates (Section 3) obtained by a bootstrapping approach. Finally, we present several applications of the arithmetization (Section 4), firstly, we give an example of a proof by computation, secondly, we prove that, given two points, we can build an equilateral triangle based on these two points in Euclidean Hilbert planes, thirdly, we show how we derived the formal proof of the axioms for the area method.

\section{Arithmetization of Geometry}

As a basis for this work, Tarski's system of geometry was chosen for its well-known metamathematical properties. We assumed the axioms for plane Euclidean geometry given in [SST83] excluding the axiom introducing continuity (Dedekind cuts). As noted before this axiom system is bi-interpretable with the theory defined by the first four groups of Hilbert's axioms. Hence, our results are also available in the context of Hilbert's axioms. Tarski's axiom system is based on a single primitive type depicting points and two predicates, namely congruence and betweenness. $A B \equiv C D$ states that the segments $A B$ and $C D$ have the same length. $A-B-C$ means that $A$, $B$ and $C$ are collinear and $B$ is between $A$ and $C$ (and $B$ may be equal to $A$ or $C$ ). 


\subsection{Definition of Arithmetic Operations}

To define the arithmetic operations, we first needed to fix the neutral element of the addition $O$ and the neutral element of the multiplication $E$. The line $O E$ will then contain all the points for which the operations are well-defined as well as their results. Moreover, a third point $E^{\prime}$ is required for the definitions of these operations. It is to be noticed that these points should not be collinear (collinearity is expressed with the Col predicate defined in Table A.1 where all the predicates which are not defined in this paper are listed together with their definition). Indeed, if they were collinear the results of these operations would not be well-defined. The three points $A$, $B$ and $C$ need to belong to line $O E$. These properties are formalized by the definition Ar2:

Definition Ar2 O E E' A B C :=

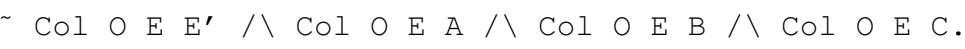

\section{Definition of Addition}

The definition of addition that we adopted is the same as in [SST83] which is expressed in terms of parallel projection. One could think of a definition of addition by extending the segment $E B$ by the segment $E A$, this would work only for points (numbers) which have the same sign. The parallel projection allows to have a definition which is correct for signed numbers. The same definition is given by Hilbert in Chapter V, Section 3 of [Hil60]. $\mathrm{P} j$ is a predicate that captures parallel projection. P $\mathrm{A} A \mathrm{~B} \quad \mathrm{C}$ D denotes that either lines $A B$ and $C D$ are parallel or $C=D$. The addition is defined as a predicate and not as a function. Sum $O \mathrm{E}^{\prime} \mathrm{E}^{\prime} \mathrm{A} B \mathrm{~B}$ means that $C$ is the sum of $A$ and $B$ wrt. $O, E$ and $E^{\prime}$.
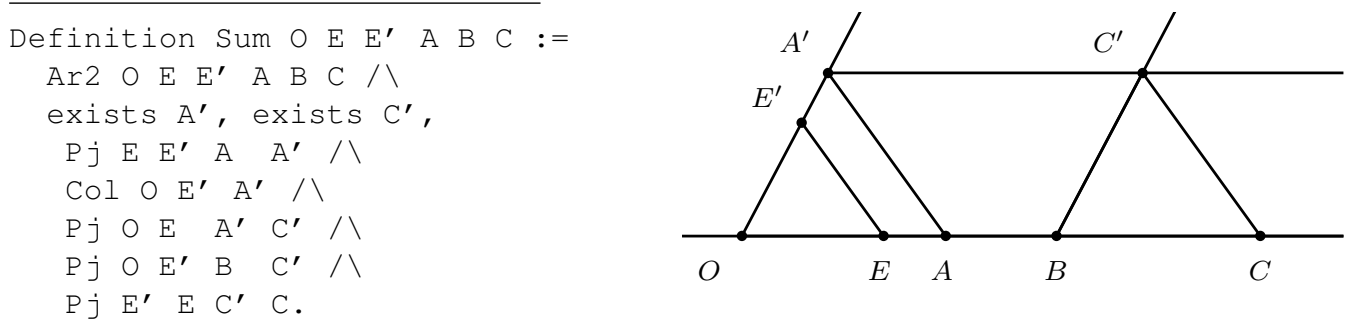

To prove existence and uniqueness of the last argument of the sum predicate, we introduced an alternative and equivalent definition highlighting the ruler and compass construction presented by Descartes. Proj P Q A B X Y states that $Q$ is the image of $P$ by projection on line $A B$ parallel to line $X Y$ and Par A B C D denotes that lines $A B$ and $C D$ are parallel.

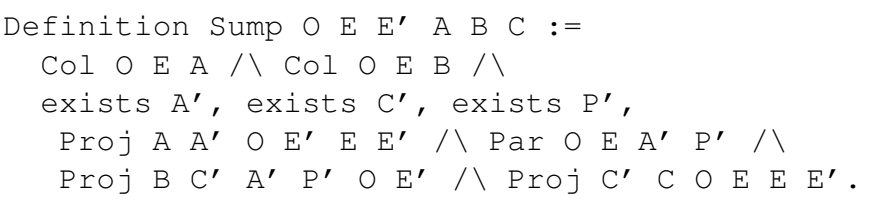

One should note that this definition is in fact independent of the choice of $E^{\prime}$, and it is actually proved in [SST83]. Furthermore, we could prove it by characterizing the sum predicates in terms of the segment congruence predicate: 


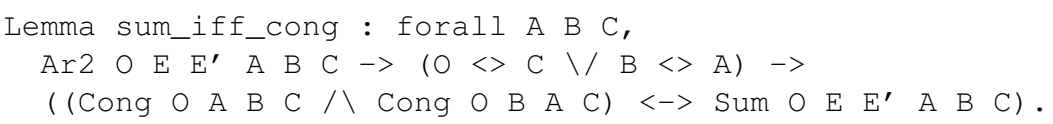

We used properties of parallelograms to prove this characterization and the properties about Sum, contrary to what is done in [SST83] where they are proven using Desargues' theorem ${ }^{1}$.

\section{Definition of Multiplication}

As for the definition of addition, the definition of multiplication presented in [SST83] uses the parallel projection:
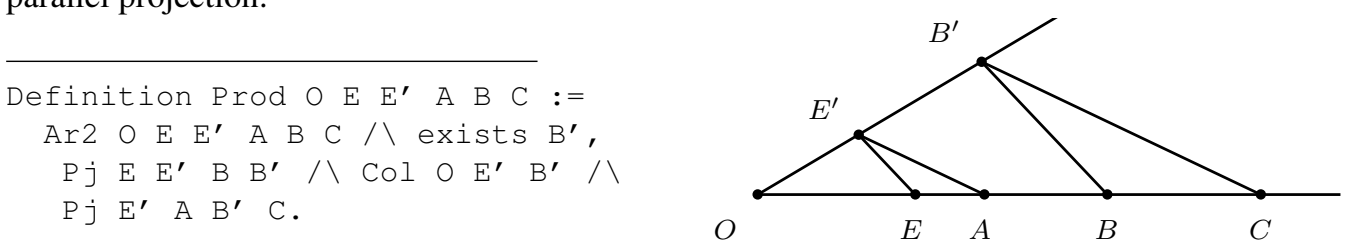

Similarly to the definition of addition, we introduced an alternative definition which underlines that the definition corresponds to Descartes' ruler and compass construction:

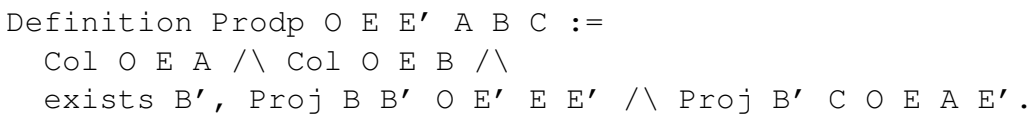

Using Pappus' theorem, we proved the commutativity of the multiplication and, using Desargues' theorem, its associativity. We omit the details of these well-known facts.

\subsection{Construction of an Ordered Field}

In his thesis [Gup65], Gupta provided an axiom system for the theory of $n$-dimensional Cartesian spaces over the class of all ordered fields. In [SST83], a $n$-dimensional Cartesian space over Pythagorean ordered fields is constructed. We restricted ourselves to the planar case.

As remarked by $\mathrm{Wu}$, the proofs are not as trivial as presented by Hilbert:

"However, the proofs are cumbersome and not always easy. They can all be found in Hilbert's 'Grundlagen der Geometrie.' It should be noted that Hilbert's proofs were only given for the generic cases, whereas the degenerate cases also need to be considered. Thus, the complete proofs are actually much more cumbersome than the original ones."

(Wen-Tsün Wu, page 40 [Wu94])

\subsubsection{Field properties}

In Tarski's system of geometry, the addition and multiplication are defined as relations capturing their semantics and afterwards the authors of [SST83] generalize these definitions to obtain total functions. Indeed, the predicates Sum and Prod only hold if the predicate Ar2 holds for the same points. All field properties are then proved geometrically [SST83]. In theory, we could carry out with the relational versions of the arithmetic operators. But in practice, this causes

\footnotetext{
1 We can remark that we proved the parallel case of this theorem without relying on Pappus' theorem but on properties about parallelograms.
} 
two problems. Firstly, the statements become quickly unreadable. Secondly, we cannot apply the standard Coq tactics ring and field because they only operate on rings and fields whose arithmetic operators are represented by functions.

Obtaining the function from the functional relation is implicit in [SST83]. In practice, in the Coq proof assistant, we employed the constructive_definite_description axiom provided by the standard library:

Axiom constructive_definite_description :

forall (A:Type) ( $\mathrm{P}: \mathrm{A}->$ Prop), (exists! $\mathrm{x}, \mathrm{P} \mathrm{x})->\{\mathrm{x}: \mathrm{A} \mid \mathrm{P} \mathrm{x}\}$.

It allows to convert any relation which is functional to a proper Coq function. Another option, would be to change our axiom system to turn the existential axioms into their constructive version. We plan to adopt this approach in the future, but for the time being, we use the constructive definite description axiom provided by the standard library. As the use of the $\epsilon$ axiom turns the intuitionistic logic of Coq into an almost classical logic [Bel93], we decided to postpone the use of this axiom as much as possible. For example, we defined the sum function relying on the following lemma ${ }^{2}$ :

Lemma sum_f : forall A B, Col O E A $\rightarrow$ Col O E B $\rightarrow$

$\left\{\mathrm{C} \mid\right.$ Sum $\left.O \mathrm{E} \mathrm{E}^{\prime} \mathrm{A} B \mathrm{C}\right\}$.

This function is not total, the sum is only defined for points which belong to our ruler $(O E)$. Nothing but total functions are allowed in Coq, hence to define the ring and field structures, we needed a dependent type (a type which depends on a proof), describing the points which belong to the ruler. In Coq's syntax it is expressed as:

Definition $\mathrm{F}:$ Type $:=\{\mathrm{P}$ : Tpoint $\mid \mathrm{Col} O \mathrm{E} \mathrm{P}\}$.

Here, we chose a different approach than in [SST83], in which, as previously mentioned, the arithmetic operations are generalized to obtain function symbols without having to restrict the domain of the operations. Doing so implies that the field properties only hold under the hypothesis that all considered points belong to the ruler. This has the advantage of enabling the use of function symbols but the same restriction to the points belonging to the ruler is needed.

We defined the equality on $\mathrm{F}$ with the standard Coq function proj1_sig which projects on the first component of our dependent pair, forgetting the proof that the points belong to the ruler:

Definition EqF (x y : F) $:=($ proj1_sig $x)=($ proj1_sig y) .

This equality is naturally an equivalence relation. One should remark that projecting on the first component is indeed needed. Actually, we have proven (together with Schreck) in [BNSB14a] that the decidability of the equality implies the decidability of every predicate present in [SST83]. The decidability that we assumed was in Prop and not in Set to avoid assuming a much stronger axiom. By Hedberg's theorem, equality proofs of types which are in Set are unique. This allows to get rid of the proof relevance for dependent types. Nevertheless the decidability of the collinearity predicate is in Prop, where equality proofs are not unique. Therefore, the proof component is not irrelevant here.

2 We chose to omit the definitions of functions corresponding to the arithmetic operations to avoid technicalities. 
Next, we built the arithmetic functions on the type F. In order to employ the standard Coq tactics ring and field or the implementation of setoids in Coq [Soz10], we proved some lemmas asserting that the operations are morphisms relative to our defined equality. For example, the fact that $A=A^{\prime}$ and $B=B^{\prime}$ (where = denotes EqF) implies $A+B=A^{\prime}+B^{\prime}$ is defined in Coq as:

Global Instance addF_morphism : Proper (EqF $==>\mathrm{EqF}==>\mathrm{EqF})$ AddF .

With a view to apply the Gröbner basis method, we also proved that $\mathrm{F}$ is an integral domain. This would seem trivial, as any field is an integral domain, but we actually proved that the product of any two non-zero elements is non-zero even before we proved the associativity of the multiplication. Indeed, in order to prove this property, one needs to distinguish the cases where some products are null from the general case. Finally, we can prove we have a field:

Lemma fieldF : field_theory OF OneF AddF MulF SubF OppF Divf InvF EqF.

We present now the formalization of the proof that the field is Pythagorean (every sum of two squares is a square). We built a function $\operatorname{Pyth}(a, b)=\sqrt{a^{2}+b^{2}}$ derived from the following PythRel relation. Note that we needed to treat some special cases separately:

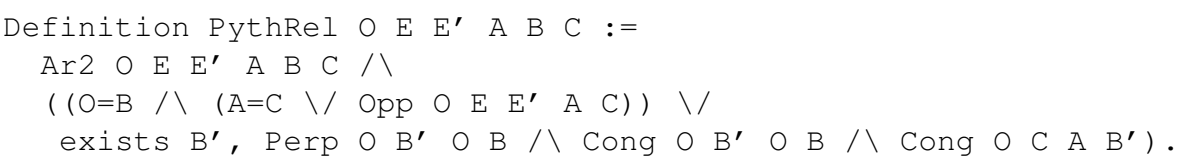

Using Pythagorean theorem (see next section), we showed that the definition of PythRel has the proper semantics $\left(A^{2}+B^{2}=C^{2}\right)$ :

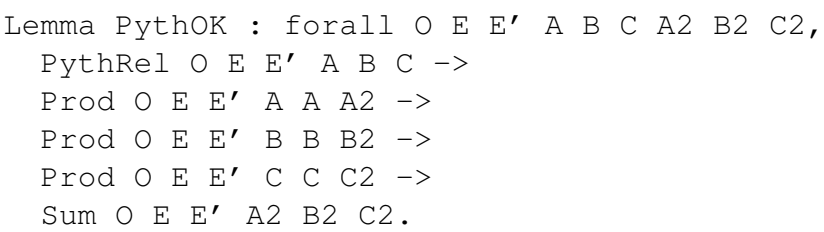

Then, we proved that if we add the assumption that the last argument of PythRel is positive then the relation is functional:

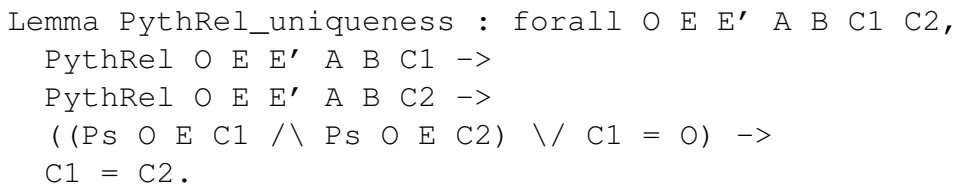

\subsubsection{Order}

We proved that $\mathrm{F}$ is an ordered field. For convenience we proved it for two equivalent definitions. Namely, that one can define a positive cone on $\mathrm{F}$ or that $\mathrm{F}$ is equipped with a total order on F which is compatible with the operations. In [SST83], one can only find the proof based on the first definition. The characterization of the betweenness predicate in [SST83] is expressed in 
terms of the order relation and not positivity. The second definition is therefore better suited for this proof than the first one. Nevertheless, for the proof relying on the second definition, we decided to prove the implication between the first and the second definition. Actually, an algebraic proof, unlike a geometric one, rarely includes tedious case distinctions.

In order to define the positive cone on $\mathrm{F}$, we needed to define positivity. A point is said to be positive when it belongs to the half-line $O E$. Out $\bigcirc \mathrm{A}$ B indicates that $O$ belongs to line $A B$ but does not belong to the segment $\overline{A B}$, or that $B$ belongs to ray $O A$.

Definition PS O E A := Out O A E.

A point is lower than another one if their difference is positive and the lower or equal relation is trivally defined. Diff $O \mathrm{E} \mathrm{E}^{\prime} \mathrm{A}$ B C denotes that $C$ is the difference of $A$ and $B$ wrt. $O$, $E$ and $E^{\prime}$

Definition LtP $O \mathrm{E} \mathrm{E}^{\prime} \mathrm{A} B:=$ exists $\mathrm{D}$, Diff $O \mathrm{E}^{\prime} \mathrm{E}^{\prime} \mathrm{B} A \mathrm{D} / \mathrm{PS} O \mathrm{E} \mathrm{D}$.

Definition LeP $O \mathrm{E}^{\prime} \mathrm{A} B:=$ LtP $O \mathrm{E} \mathrm{E}^{\prime} \mathrm{A} \mathrm{B} \backslash / \mathrm{A}=\mathrm{B}$.

The lower or equal relation is then shown to be a total order compatible with the arithmetic operations.

\section{Algebraic Characterization of Geometric Predicates}

It is well-known that having algebraic characterizations of geometric predicates is very useful. Indeed, if we know a quantifier-free algebraic characterization for every geometric predicate present in the statement of a lemma, the proof can then be seen as verifying that the polynomial(s) corresponding to the conclusion of the lemma belong(s) to the radical of the ideal generated by the polynomials corresponding to the hypotheses of the lemma. Since there are computational ways (for example, the Gröbner basis method) to do this verification, these characterizations allow to obtain proofs by computations. In this section, we present our formalization of the coordinatization of geometry and the method we employed to automate the proofs of algebraic characterizations.

\subsection{Coordinatization of Geometry}

To define coordinates, we first defined what is a proper orthonormal coordinate system (Cs) as an isosceles right triangle for which the length of the congruent sides equals the unity. Per $A$ B $C$ states that $A, B$ and $C$ form a right triangle.

Definition CS O E S U1 U2 :=

$\mathrm{O} \ll \mathrm{E} / \backslash$ Cong $\mathrm{O} \mathrm{E} \mathrm{S} \mathrm{U} 1 /$ Cong $\mathrm{O} \mathrm{E} \mathrm{S}$ U2 $\bigwedge$ Per U1 S U2.

The predicate $\mathrm{Cd} O \mathrm{E} \quad \mathrm{S} \quad \mathrm{U} 1 \mathrm{U} 2 \mathrm{P} \quad \mathrm{X}$ Y denotes that the point $P$ has coordinates $X$ and $Y$ in the coordinate system $\mathrm{CS} O \mathrm{E}$ S U1 U2. Cong_3 A B C D E F designates that the triangles $A B C$ and $D E F$ are congruent and Projp P $Q$ A B means that $Q$ is the foot of the perpendicular from $P$ to line $A B$.

Definition Cd O E S U1 U2 P X Y :=

Cs O E S U1 U2 八 Coplanar P S U1 U2 八 
(exists PX, Projp P PX S U1 八 Cong_3 O E X S U1 PX) 八

(exists PY, Projp P PY S U2 / Cong_3 O E Y S U2 PY).

According to Borsuk and Szmielew [BS60], in planar neutral geometry (i.e. without assuming the parallel postulate) it cannot be proved that the function associating coordinates to a given point is surjective. But assuming the parallel postulate, we can show that there is a one-to-one correspondence between the pairs of points on the ruler representing the coordinates and the points of the plane:

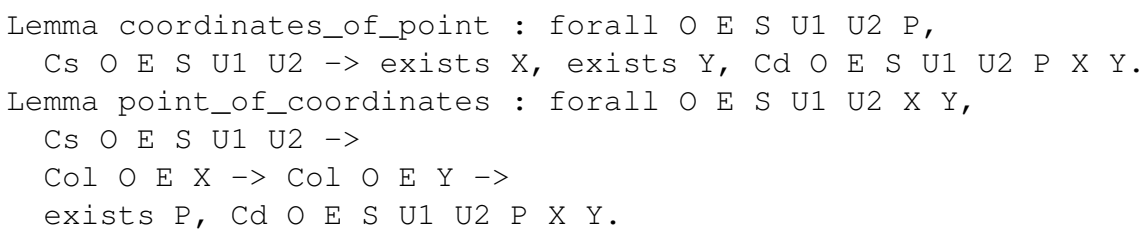

\subsection{Algebraic Characterization of Congruence}

We recall that Tarski's system of geometry has two primitive relations: congruence and betweenness. Following Schwabhäuser, we formalized the characterizations of these two geometric predicates. We have shown that the congruence predicate which is axiomatized is equivalent to the usual algebraic formula stating that the squares of the Euclidean distances are equal ${ }^{3}$ :

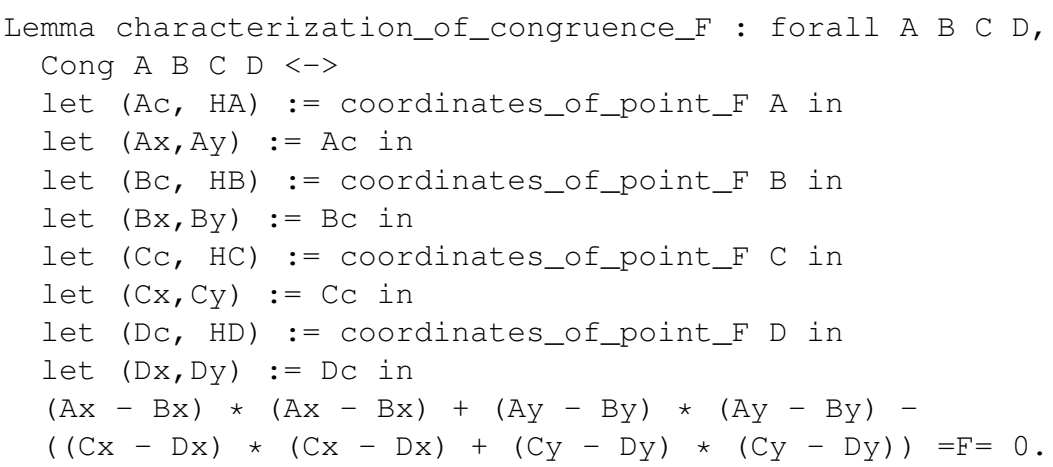

The proof relies on Pythagoras' theorem (also known as Kou-Ku theorem). Note that we need a synthetic proof here. It is important to notice that we cannot use an algebraic proof because we are building the arithmetization of geometry. The statements for Pythagoras' theorem that have been proved previously ${ }^{4}$ are theorems about vectors: the square of the norm of the sum of two orthogonal vectors is the sum of the squares of their norms. Here we provide the formalization of the proof of Pythagoras' theorem in a geometric context. Length $O E E^{\prime} A$ B L expresses that the length of segment $A B$ can be represented by a point called $L$ in the coordinate system $O, E, E^{\prime}$.

\footnotetext{
3 In the statement of this lemma, coordinates_of_point_F asserts the existence for any point of corresponding coordinates in $\mathrm{F}^{2}$ and the arithmetic symbols denote the operators or relations according to the usual notations.

4 A list of statements of previous formalizations of Pythagoras' theorem can be found on Freek Wiedijk web page: http://www.cs.ru.nl/ freek/100/.
} 


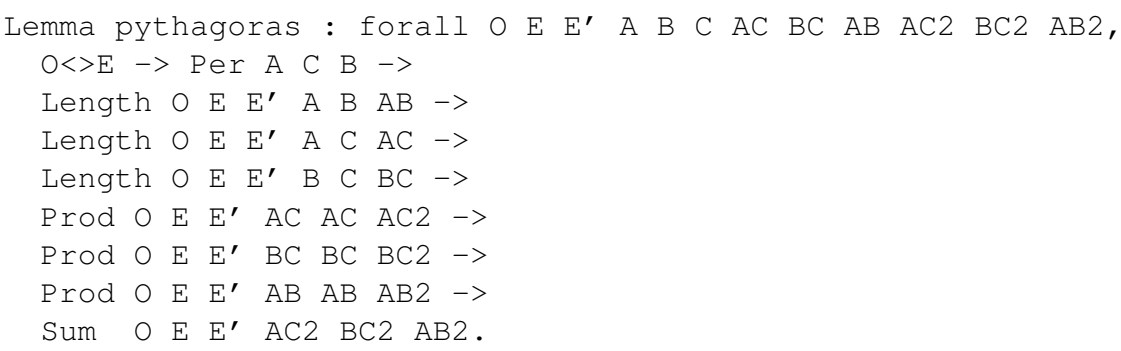

Our formal proof of Pythagoras' theorem itself employs the intercept theorem (also known in France as Thales' theorem). These last two theorems represent important theorems in geometry, especially in the education. Prodg $O E_{E}^{\prime}$ A B C designates the generalization of the multiplication which establishes as null the product of points for which Ar2 does not hold.

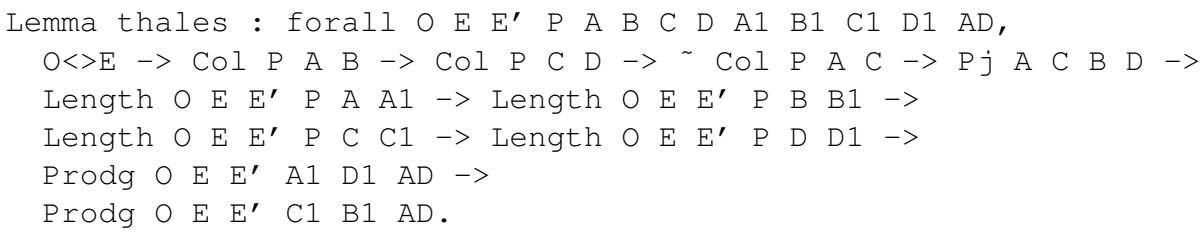

\subsection{Automated Proofs of the Algebraic Characterizations}

In this subsection, we present our formalization of the translation of a geometric statement into algebra adopting the usual formulas as shown on Table 1 . In this table we denoted by $x_{P}$ the abscissa of a point $P$ and by $y_{P}$ its ordinate.

To obtain the algebraic characterizations of the others geometric predicates we adopted an original approach based on bootstrapping: we applied the Gröbner basis method to prove the algebraic characterizations of some geometric predicates which will be used in the proofs of the algebraic characterizations of other geometric predicates. The trick consists in a proper ordering of the proofs of the algebraic characterizations of geometric predicates relying on previously characterized predicates.

For example, we characterized parallelism in terms of midpoints and collinearity using the famous midpoint theorem that we previously proved synthetically ${ }^{5}$. Midpoint M A B means that $M$ is the midpoint of $A$ and $B$.

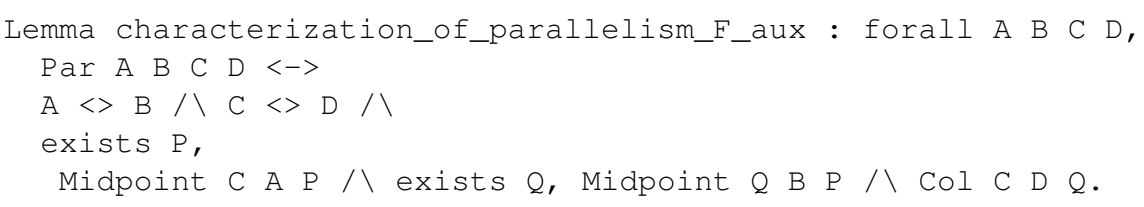

In the end, we only proved the characterizations of congruence, betweenness, equality and collinearity manually. The first three were already present in [SST83] and the last one was fairly

\footnotetext{
5 Note that it is important that we have a synthetic proof, because we cannot use an algebraic proof to obtain the characterization of parallelism since an algebraic proof would depend on the characterization of parallelism.
} 


\begin{tabular}{|c|c|}
\hline Geometric predicate & Algebraic Characterization \\
\hline \multirow[t]{3}{*}{$A=B$} & $x_{A}-x_{B}=0 \wedge$ \\
\hline & $y_{A}-y_{B}=0$ \\
\hline & or $\left(x_{A}-x_{B}\right)^{2}+\left(y_{A}-y_{B}\right)^{2}=0$ \\
\hline$A B \equiv C D$ & $\left(x_{A}-x_{B}\right)^{2}+\left(y_{A}-y_{B}\right)^{2}-\left(\left(x_{C}-x_{D}\right)^{2}+\left(y_{C}-y_{D}\right)^{2}\right)=0$ \\
\hline \multirow[t]{2}{*}{$A-B-C$} & $\exists t, 0 \leq t \leq 1 \wedge t\left(x_{C}-x_{A}\right)=x_{B}-x_{A} \wedge$ \\
\hline & $t\left(y_{C}-y_{A}\right)=y_{B}-y_{A}$ \\
\hline $\mathrm{Col} A B C$ & $\left(x_{A}-x_{B}\right)\left(y_{B}-y_{C}\right)-\left(y_{A}-y_{B}\right)\left(x_{B}-x_{C}\right)=0$ \\
\hline \multirow[t]{2}{*}{$A+I+B$} & $2 x_{I}-\left(x_{A}+x_{B}\right)=0 \wedge$ \\
\hline & $2 y_{I}-\left(y_{A}+y_{B}\right)=0$ \\
\hline$\triangle A B C$ & $\left(x_{A}-x_{B}\right)\left(x_{B}-x_{C}\right)+\left(y_{A}-y_{B}\right)\left(y_{B}-y_{C}\right)=0$ \\
\hline \multirow[t]{3}{*}{$A B \perp C D$} & $\left(x_{A}-x_{B}\right)\left(y_{C}-y_{D}\right)-\left(y_{A}-y_{B}\right)\left(x_{C}-x_{D}\right)=0 \wedge$ \\
\hline & $\left(x_{A}-x_{B}\right)\left(x_{A}-x_{B}\right)+\left(y_{A}-y_{B}\right)\left(y_{A}-y_{B}\right) \neq 0 \wedge$ \\
\hline & $\left(x_{C}-x_{D}\right)\left(x_{C}-x_{D}\right)+\left(y_{C}-y_{D}\right)\left(y_{C}-y_{D}\right) \neq 0$ \\
\hline \multirow[t]{3}{*}{$A B \| C D$} & $\left(x_{A}-x_{B}\right)\left(x_{C}-x_{D}\right)+\left(y_{A}-y_{B}\right)\left(y_{C}-y_{C}\right)=0 \wedge$ \\
\hline & $\left(x_{A}-x_{B}\right)\left(x_{A}-x_{B}\right)+\left(y_{A}-y_{B}\right)\left(y_{A}-y_{B}\right) \neq 0 \wedge$ \\
\hline & $\left(x_{C}-x_{D}\right)\left(x_{C}-x_{D}\right)+\left(y_{C}-y_{D}\right)\left(y_{C}-y_{D}\right) \neq 0$ \\
\hline
\end{tabular}

Table 1. Algebraic Characterizations of geometric predicates.

straightforward to formalize from the characterization of betweenness. However, it is impossible to obtain the characterizations of collinearity from the characterizations of betweenness by bootstrapping. Indeed, only a characterization of a geometric predicate $G$ with subject $\bar{x}$ of the form $G(\bar{x}) \Leftrightarrow \bigwedge_{k=1}^{n} P_{k}(x)=0 \wedge \bigwedge_{k=1}^{m} Q_{k}(x) \neq 0$ for some $m$ and $n$ and for some polynomials $\left(P_{k}\right)_{1 \leq k \leq n}$ and $\left(Q_{k}\right)_{1 \leq k \leq m}$ in the coordinates $x$ of the points can be handled by either Wu's method or Gröbner basis method. Nevertheless, in theory, the characterization of the betweenness predicate could be employed by methods such as the quantifier elimination algorithm for real closed fields formalized by Cohen and Mahboubi in [CM12]. Then we obtained automatically the characterizations of midpoint, right triangles, parallelism and perpendicularity (in this order). The characterization of midpoint can be easily proven from the fact that if a point is equidistant from two points and collinear with them, either this point is their midpoint or these two points are equal. To obtain the characterization of right triangles, we exploited its definition which only involves midpoint and segment congruence. One should notice that the existential quantifier can be handled using a lemma asserting the existence of the symmetric of a point with respect to another one. To obtain the characterization of perpendicularity, we employed the characterizations of parallelism, equality and right triangle. The characterization of parallelism 
is used to produce the intersection point of the perpendicular lines which is needed as the definition of perpendicular presents an existential representing this point. Proving that the lines are not parallel allowed us to avoid producing the point of intersection by computing its coordinates. This was more convenient as these coordinates cannot be expressed as a polynomial but only as a rational function. Having a proof in Coq highlights the fact that the usual characterizations include degenerate cases. For example, the characterization of perpendicularity in Table 1 entails that lines $A B$ and $C D$ are non-degenerate.

\section{Applications}

To demonstrate that the arithmetization of geometry is useful in practice, we describe in this section some applications. In Section 4.1, we give an example of an automatic proof using an algebraic method. In Section 4.2, we use the language of vectors defined using coordinates to derive the axioms for another automated deduction method: the area method. In Section 4.3, we solve a challenge proposed by Beeson: we prove that equilateral triangles can be constructed without any line-circle continuity axiom.

\subsection{An Example of a Proof by Computation}

To show that the arithmetization of geometry is useful in practice, we applied the nsat $z$ tactic developed by Grégoire, Pottier and Théry [GPT11] to one example. This tactic corresponds to an implementation of the Gröbner basis method. Our example is the nine-point circle theorem (Fig. 1) which states that the following nine points are concyclic: the midpoints of each side of the triangle, the feet of each altitude and the midpoints of the segments from each vertex of the triangle to the orthocenter ${ }^{6}$ :

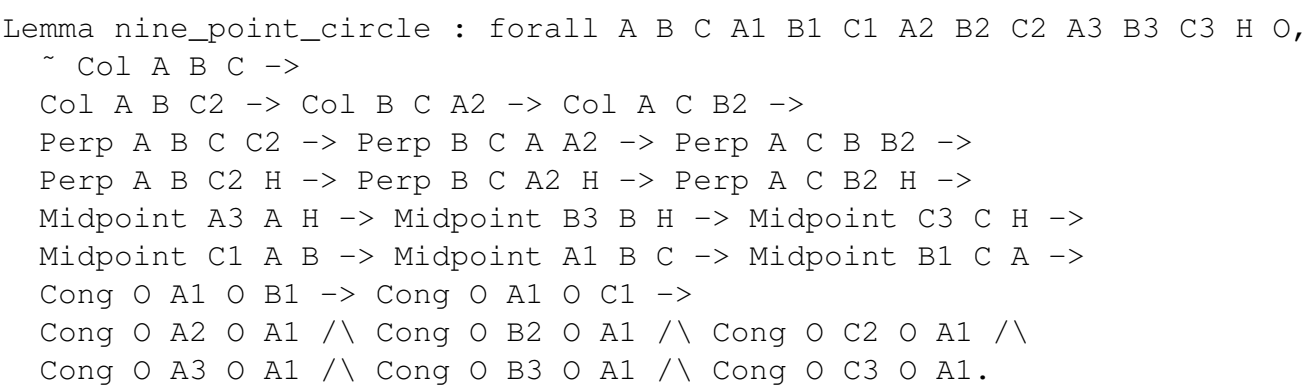

Compared to other automatic proofs using purely algebraic methods (either Wu's method or Gröbner basis method), the statement that we proved is syntactically the same, but the definitions and axioms are completely different. We did not prove a theorem about polynomials but a geometric statement. This proves that the nine-point circle theorem is true in any model of Tarski's Euclidean plane geometry axioms (without continuity) and not only in a specific one. We should remark that to obtain the proof automatically with the nsatz tactic, we had to clear the hypotheses that the lines appearing as arguments of the Perp predicate are well-defined. In theory, this should only render the problem more difficult to handle, but in practice the nsat $z$ tactic can only solve the problem without these additional (not needed) assumptions. Non-degeneracy

\footnotetext{
6 In fact, many well-known points belong to this circle and this kind of properties can easily be proved formally using barycentric coordinates [NB16].
} 


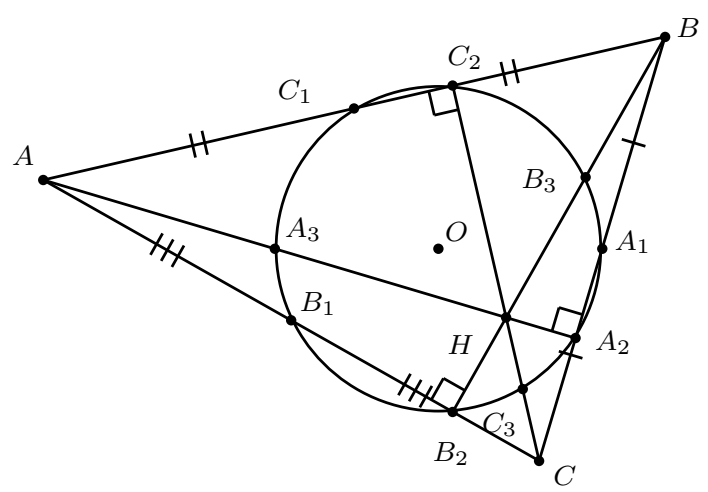

Fig. 1. Euler's nine-point circle

conditions represent an issue as often in geometry. Here we have an example of a theorem where they are superfluous but, while proving the characterizations of the geometric predicates, they were essential.

Moreover, we should notice that Wu's or the Gröbner basis methods rely on the Nullstellensatz and are therefore only complete in an algebraically closed field. Hence, we had to pay attention to the characterization of equality. Indeed, as the field $\mathrm{F}$ is not algebraically closed, one can prove that $x_{A}=x_{B}$ and $y_{A}=y_{B}$ is equivalent to $\left(x_{A}-x_{B}\right)^{2}+\left(y_{A}-y_{B}\right)^{2}=0$ but this is not true in an algebraically closed one. Therefore, the tactic nsat $z$ is unable to prove this equivalence.

\subsection{Connection with the Area Method}

The area method, proposed by Chou, Gao and Zhang in the early 1990's, is a decision procedure for a fragment of Euclidean plane geometry [CGZ94]. It can efficiently prove many nontrivial geometry theorems and produces proofs that are often very concise and human-readable.

The method does not use coordinates and instead deals with problems stated in terms of sequences of specific geometric construction steps and the goal is expressed in terms of specific geometric quantities:

(1) ratios of lengths of parallel directed segments,

(2) signed areas of triangles,

(3) Pythagoras difference of points (for the points $A, B, C$, this difference is defined as $P y(A B C)=\overline{A B}^{2}+\overline{B C}^{2}-\overline{A C}^{2}$.

In a previous work, the third author together with Janičić and Quaresma have formalized in Coq the area method based on a variant of the axiom system used by Chou, Gao and Zhang [JNQ12]. Our axiom system was based on the concept of signed distance instead of ratio of signed distance. The axioms of our formalization of the area method are listed on Table 2. This allows to deduce some properties of ratios from the field axioms. For example, one can deduce from the field axioms that $\frac{\overline{A B}}{\overline{C D}} \frac{\overline{C D}}{\overline{E F}}=\frac{\overline{A B}}{\overline{E F}}$.

\subsubsection{Definition of the geometric quantities}

The first step toward proving the axioms of the area method is to define the geometric quantities in the context of Tarski's axioms.

First, we defined the usual operations on vectors, cross and scalar products: 
Definition vect $:=(F * F)$ otype.

Definition cross_product ( $\mathrm{u} v$ : vect) : $\mathrm{F}$ :=

fst $u$ * snd $v-$ snd $u$ * fst $v$.

Definition scalar_product ( $\mathrm{U} v$ : vect) $: \mathrm{F}:=$

fst $u$ * fst $v+$ snd $u *$ snd $v$.

Then, the ratio of signed distances can be defined using a formula provided by Chou, Gao and Zhang. This formula has the advantage to give a definition which is a total function:

$$
\frac{\overline{A B}}{\overline{C D}}=\frac{\overrightarrow{A B} \cdot \overrightarrow{C D}}{\overrightarrow{C D} \cdot \overrightarrow{C D}}
$$

In Coq's syntax:

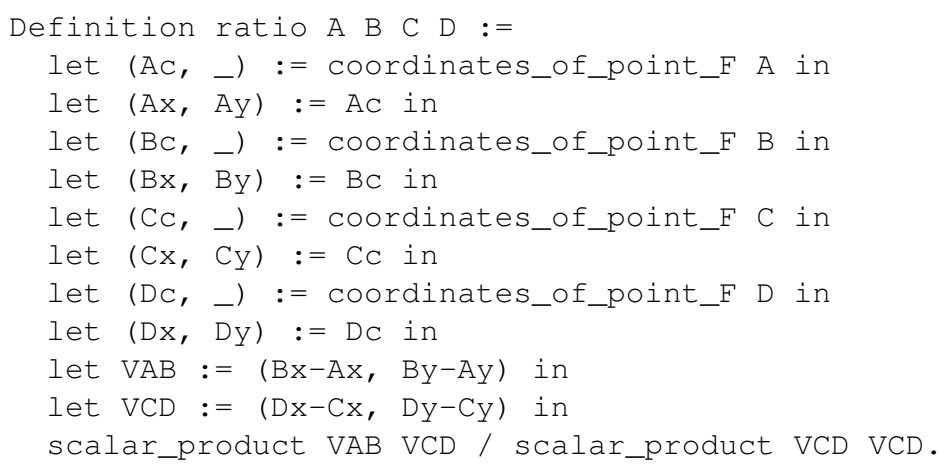

For the signed area, we used the cross product:

$$
\mathcal{S}_{A B C}=\frac{1}{2} \overrightarrow{A B} \times \overrightarrow{A C}
$$

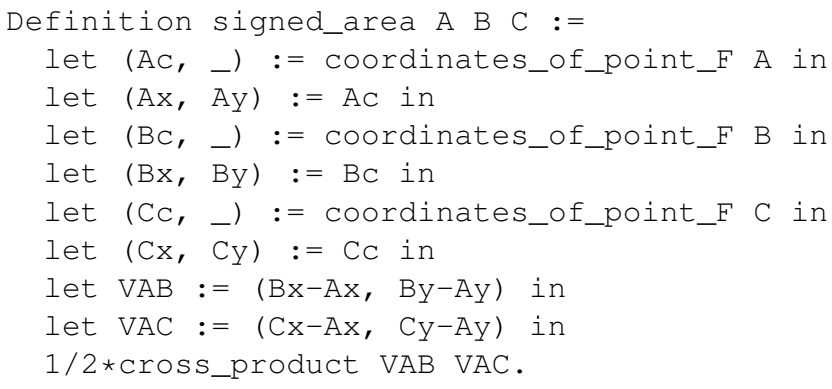

Note that in the formal proof, we also used the concept of twice the signed area, because it does not change the ratio of areas, nor the equality between areas but it simplifies the proofs.

To define the Pythagoras difference, we used the square of the signed distance:

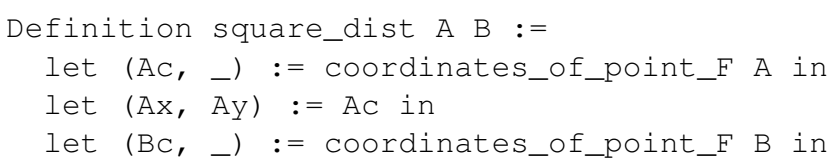




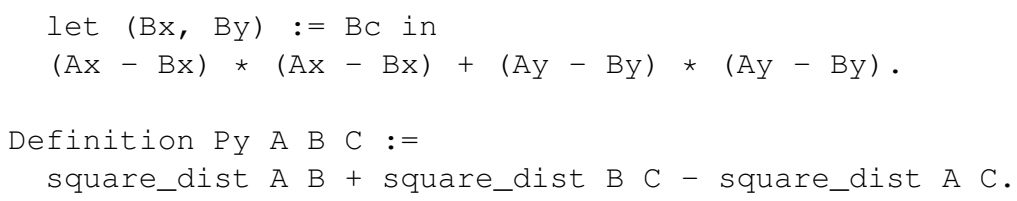

\subsubsection{Proofs of the axioms}

In this section, we demonstrate that the assertion, made previously on page 8 of [JNQ12] which stated that all the axioms of the area method can be derived from Hilbert's axioms, is indeed correct. In this previous work, we stated that proving the area method axioms from Hilbert's axioms would be cumbersome, but thanks to the arithmetization of geometry and algebraic methods for automated deduction in geometry, we can now obtain the proofs of the axioms of the area method quite easily. This shows the strength of using a proof assistant, allowing both synthetic and algebraic proofs together with automated deduction. We believe that mixing synthetic and algebraic proof is very powerful and can have several applications. Indeed, it has been demonstrated recently by Mathis and Schreck. They have resolved open geometric construction problems using a combination of algebraic computation with a synthetic approach [MS16].

The first axiom $A M_{1}$ is a direct consequence of the characterization of point equality described in Section 3.3. The axioms $A M_{2}, A M_{3}$ and $A M_{6}$ are trivial. One only needs to prove that two polynomials are equal, which can be done automatically in most, if not all, of the proof assistants. In Coq, it can be done employing the ring tactic. Notice that a variant of the axiom $A M_{4}$ can be proved for ratios even without the assumption that $A, B$ and $C$ are collinear:

Lemma chasles_ratios : forall A B C P Q

$\mathrm{P}<>\mathrm{Q}->$ ratio A B P $\mathrm{Q}$ +ratio B C P Q $=$ F= ratio A C P $\mathrm{Q}$.

Axiom $A M_{5}$ is a direct consequence of Tarski's lower dimensional axiom (or the corresponding Hilbert's axiom). For the proof of axiom $A M_{7}$, we gave explicitly the coordinates of the point $P$ asserted to exist by this axiom. The axioms $A M_{8}, A M_{9}, A M_{10}$ and $A M_{13}$ can be proved using the implementation of Gröbner basis method in Coq. For the axioms $A M_{11}$ and $A M_{12}$, the implementation of Gröbner basis method in Coq failed. We had to find another solution. We first proved the equivalence between the definition of the parallelism and perpendicularity predicates using areas and the geometric definition. Indeed, in the area method axioms, collinearity and parallelism are defined using the signed area and perpendicularity using the Pythagoras difference:

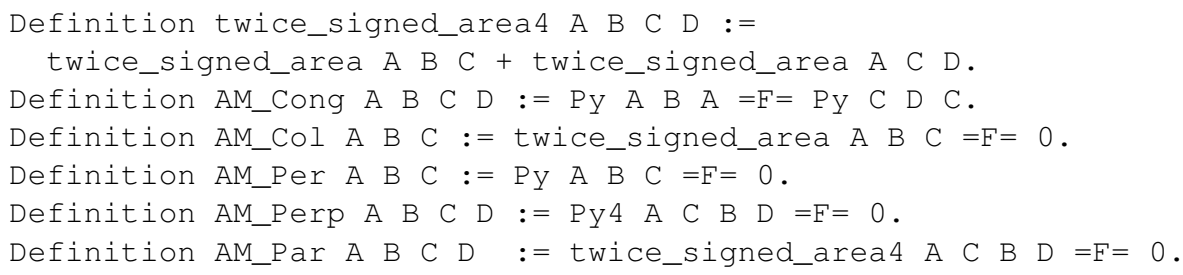

Then we proved the equivalence with the geometric definitions:

Lemma Cong_AM_Cong: forall A B C D, AM_Cong A B C D $<->$ Cong A B C D.

Lemma Col_AM_Col : forall A B C, AM_Col A B C $<->$ Col A B C.

Lemma Per_AM_Per : forall A B C, AM_Per A B C $<->$ Per A B C. 


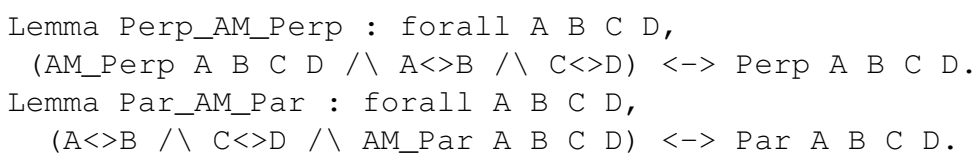

Finally, we could prove the axioms $A M_{11}$ and $A M_{12}$ which correspond to properties which have already been proved in the context of Tarski's axioms. The fact that we have the possibility to perform both automatic theorem proving and interactive theorem proving in the same setting is very useful: it allows to perform manual geometric reasoning when the algebraic method fails and to get some proofs automatically when it is possible.

$A M_{1} \overline{A B}=0$ if and only if the points $A$ and $B$ are identical.

$A M_{2} \quad \mathcal{S}_{A B C}=\mathcal{S}_{C A B}$

$A M_{3} \quad \mathcal{S}_{A B C}=-\mathcal{S}_{B A C}$

$A M_{4}$ If $\mathcal{S}_{A B C}=0$ then $\overline{A B}+\overline{B C}=\overline{A C}$ (Chasles' axiom).

$A M_{5}$ There are points $A, B, C$ such that $\mathcal{S}_{A B C} \neq 0$ (dimension; not all points are collinear).

$A M_{6} \mathcal{S}_{A B C}=\mathcal{S}_{D B C}+\mathcal{S}_{A D C}+\mathcal{S}_{A B D}$ (dimension; all points are in the same plane)

$A M_{7}$ For each element $r$ of $F$, there exists a point $P$, such that $\mathcal{S}_{A B P}=0$ and $\overline{A P}=r \overline{A B}$ (construction of a point on the line).

$A M_{8}$ If $A \neq B, \mathcal{S}_{A B P}=0, \overline{A P}=r \overline{A B}, \mathcal{S}_{A B P^{\prime}}=0$ and $\overline{A P^{\prime}}=r \overline{A B}$, then $P=P^{\prime}$ (uniqueness).

$A M_{9}$ If $P Q \| C D$ and $\frac{\overline{P Q}}{\overline{C D}}=1$ then $D Q \| P C$ (parallelogram).

$A M_{10}$ If $\mathcal{S}_{P A C} \neq 0$ and $\mathcal{S}_{A B C}=0$ then $\frac{\overline{A B}}{\overline{A C}}=\frac{\mathcal{S}_{P A B}}{\mathcal{S}_{P A C}}$ (proportions).

$A M_{11}$ If $C \neq D$ and $A B \perp C D$ and $E F \perp C D$ then $A B \| E F$.

$A M_{12}$ If $A \neq B$ and $A B \perp C D$ and $A B \| E F$ then $E F \perp C D$.

$A M_{13}$ If $F A \perp B C$ and $\mathcal{S}_{F B C}=0$ then $4 \mathcal{S}_{A B C}^{2}=\overline{A F}^{2} \overline{B C}^{2}$ (area of a triangle).

Table 2. The axiom system for the area method

\subsection{Equilateral triangle construction in Euclidean Hilbert's planes}

In this section, we solve a challenge proposed by Beeson in [Bee13]: we obtain a mechanized proof that given two points $A$ and $B$ we can always construct an equilateral triangle based on these two points without any continuity axiom. This is the first proposition of the first book of Euclid's Elements [EHD02], but Euclid's proof assumes implicitly the axiom of line-circle continuity, which states that the intersections of a line and a circle exist under some conditions. Assuming line-circle continuity, the proof is straightforward. The challenge is to complete the proof without any continuity axiom. It is possible to prove that such a triangle exists in any Euclidean Hilbert plane. But Pambuccian has shown that this theorem can not be proved in all Hilbert 
planes, even if one assumes Bachmann's Lotschnittaxiom or the Archimedes' axiom [Pam98]. The proof is based on Pythagoras' theorem, and, as we now have access to automatic deduction in geometry using algebraic methods, the theorem can be proved automatically. Let $a$ be the distance $A B$, we need to construct the length $\frac{\sqrt{3}}{2} a$. It is easy to reproduce the construction proposed by Hilbert shown on Figure 2: $P$ is a point on the perpendicular to $A B$ through $B$ such that $A B \equiv B P, Q$ is a point on the perpendicular to $A P$ through $P$ such that $A B \equiv P Q, R$ is the midpoint of segment $A Q, I$ is the midpoint of the segment $A B, C$ is finally constructed as a point on the perpendicular to $A B$ through $I$ such that $I C \equiv A R$. The fact that the midpoint can be constructed without any continuity axiom is non-trivial but we have already formalized Gupta's proof [Gup65, Nar07]. The proof that the whole construction is correct is a consequence of two applications of Pythagoras' theorem but it can be obtained automatically using the Gröbner basis method. Note again that the combination of interactive and automatic reasoning was crucial. We could construct the point by hand and use the automatic prover to check that the construction is correct.

Lemma exists_equilateral_triangle : forall A B, exists $\mathrm{C}$, Cong A B A C $\backslash$ Cong A B B C.

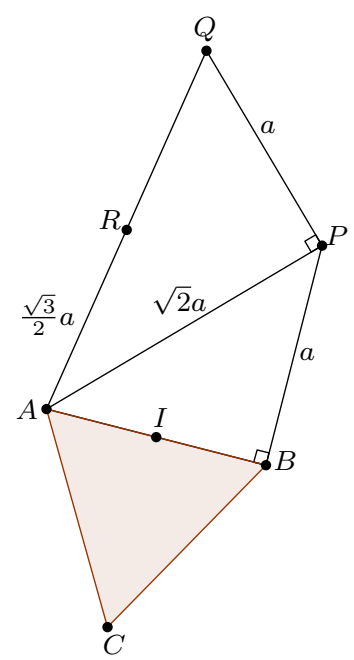

Fig. 2. Construction of an equilateral triangle on the base $A B$ without line-circle intersection.

\section{Conclusion}

In this paper, we produced the first synthetic and formal proofs of the intercept and Pythagoras' theorems. Furthermore, we obtained the arithmetization of geometry in the Coq proof assistant. This completes the formalization of the two-dimensional results contained in part one of [SST83]. To obtain the algebraic characterizations of some geometric predicates, we adopted an original approach based on bootstrapping. Our formalization of the arithmetization of geometry paves the way for the use of algebraic automated deduction methods in synthetic geometry within the Coq proof assistant. Figure 3 provides an overview of the links we formalized between the different axiom systems. Note that, even if this formalization allows to use algebraic 


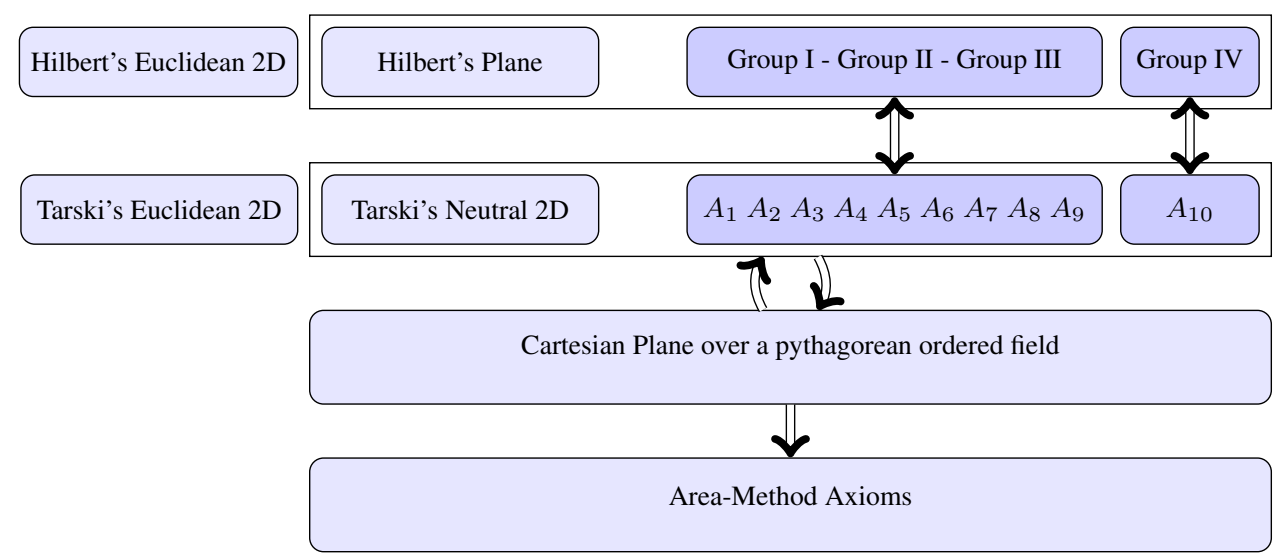

Fig. 3. Overview of the links between the axiom systems

automatic theorem provers to prove theorems assuming synthetic axioms, we cannot obtain in practice a synthetic proof by this method. Indeed, even if it is possible in principle, the synthetic proof that we would obtain by translating the algebraic computations would not be readable. In a different but similar context, Mathis and Schreck have translated by hand an algebraic solution to a geometric construction problem [MS16]. The construction obtained works. It can even be executed by GeoGebra but it is not the kind of construction that a geometer would expect.

\section{Statistics}

In a formalization effort, it is always interesting to know the value of the so-called De Bruijn factor. This factor is defined as the ratio between the size of the formalization and of the informal proof. This number is difficult to define precisely. Actually the length of the formalization depends heavily on the style of the author, and can vary in a single formalization. Likewise the length of the textbook proof fluctuates a lot depending on the author. For example, one can see below that Hilbert's description of the arithmetization of geometry is much shorter than the one produced by Schwabhäuser. Moreover, during our formalization effort, we noticed that the De Bruijn factor is not constant in a single book. Indeed, the proofs in the first chapters of [SST83] are more precise than the last chapters which leave more room for implicit arguments and cases.

The GeoCoq library currently consists (as of Nov 2015) of about 2800 lemmas and more than 100,000 lines of code. In Table 3, we provide statistics about the part of the development described in this paper. We compare the size of the formalization to the number of pages in the two books [SST83] and [Hil60]. Our formalization follows mainly [SST83], however, we proved some additional results about the characterization of geometric predicates. The proofs of these characterizations can be found in [Wu94], but the lengths of the formal and informal proofs cannot really be compared, because we proved these characterizations mainly automatically (see Section 3.3). Actually, we first proved the characterization of the midpoint predicate manually and afterward we realized that it can be proved automatically. The script of the proof by computation was eight times shorter than our original one.

\section{Perspectives}

A simple extension of this work would be to define square root geometrically following Descartes. This definition will require an axiom of continuity, such as line-circle continuity. 


\begin{tabular}{|c|c|c|c|c|c|c|}
\hline & \multicolumn{2}{|c|}{ Coq formalization } & \multicolumn{2}{|c|}{ [SST83] } & \multicolumn{2}{|c|}{ [Hil60] } \\
\hline & \#lemmas & \#loc & Chapter & \#pages & Chapter & \#pages \\
\hline $\begin{array}{l}\text { Construction of } \\
\text { an ordered field: }\end{array}$ & & & Ch. 14 & 17 & Ch. V.3 & 8 \\
\hline Sum & 100 & 4646 & & & & \\
\hline Product & 50 & 3310 & & & & \\
\hline Order & 38 & 1944 & & & & \\
\hline Length of segments & 39 & 3212 & Ch. 15 & 3 & - & - \\
\hline $\begin{array}{l}\text { Coordinates and } \\
\text { some characterizations }\end{array}$ & 33 & 2494 & Ch. 16 & 9 & - & - \\
\hline $\begin{array}{l}\text { Instantiation of } f i e l d F \\
\text { and other characterizations }\end{array}$ & 46 & 1206 & - & - & - & - \\
\hline Total & & 16812 & & 29 & & 8 \\
\hline
\end{tabular}

Table 3. Comparison of the length of the formal proof and the original proofs as presented by Hilbert and Schwabhäuser.

A more involved extension of this work consists in verifying the constructive version of the arithmetization of geometry introduced by Beeson in [Bee15]. This would necessitate to remove our axiom of decidability of equality and to replace it with Markov's principle for congruence and betweenness. We will have to reproduce Beeson's importation of the negative theorems present in [SST83] by implementing the Gödel double-negation interpretation and to formalize Beeson's proofs of existential theorems. Finally, to recover all ordered field properties, we will have to prove that Beeson's definitions of addition and multiplication are equivalent to the definitions presented in this paper, in the sense that they produce the same points (but without performing case distinctions).

Finally, another possible extension of this work is the formalization of the arithmetization of hyperbolic geometry. For this goal we could reuse the large portion of our formalization which is valid in neutral geometry.

Availability The Coq development is available in the GeoCoq library:

http://geocoq.github.io/GeoCoq/

\section{References}

[BBN16] Gabriel Braun, Pierre Boutry, and Julien Narboux. From Hilbert to Tarski. In Eleventh International Workshop on Automated Deduction in Geometry, Proceedings of ADG 2016, page 19, Strasbourg, France, June 2016. https://hal. inria.fr/hal-01332044.

[Bee13] Michael Beeson. Proof and computation in geometry. In Tetsuo Ida and Jacques Fleuriot, editors, Automated Deduction in Geometry (ADG 2012), volume 7993 of Lecture Notes in Artificial Intelligence, pages 1-30, Heidelberg, 2013. Springer. 
[Bee15] Michael Beeson. A constructive version of Tarski's geometry. Annals of Pure and Applied Logic, 166(11):1199-1273, 2015.

[Be193] John L Bell. Hilbert's $\epsilon$-operator in intuitionistic type theories. Mathematical Logic Quarterly, 39(1):323-337, 1993.

[BGNS17] Pierre Boutry, Charly Gries, Julien Narboux, and Pascal Schreck. Parallel postulates, excluded middle and continuity axioms: a mechanized study using Coq. https://hal.inria.fr/hal-01178236, in revision, March 2017.

$\left[\mathrm{BHJ}^{+}{ }^{15]}\right.$ Francisco Botana, Markus Hohenwarter, Predrag Janičić, Zoltán Kovács, Ivan Petrović, Tomás Recio, and Simon Weitzhofer. Automated Theorem Proving in GeoGebra: Current Achievements. Journal of Automated Reasoning, 55(1):39-59, 2015.

[Bir32] George D Birkhoff. A set of postulates for plane geometry, based on scale and protractor. Annals of Mathematics, pages 329-345, 1932.

[BN12] Gabriel Braun and Julien Narboux. From Tarski to Hilbert. In Tetsuo Ida and Jacques Fleuriot, editors, Post-proceedings of Automated Deduction in Geometry 2012, volume 7993 of LNCS, pages 89-109, Edinburgh, United Kingdom, September 2012. Jacques Fleuriot, Springer.

[BNS15] Pierre Boutry, Julien Narboux, and Pascal Schreck. A reflexive tactic for automated generation of proofs of incidence to an affine variety. https://hal.inria. fr/hal-01216750, unpublished, October 2015.

[BNSB14a] Pierre Boutry, Julien Narboux, Pascal Schreck, and Gabriel Braun. A short note about case distinctions in Tarski's geometry. In Francisco Botana and Pedro Quaresma, editors, Proceedings of the 10th Int. Workshop on Automated Deduction in Geometry, volume TR 2014/01 of Proceedings of ADG 2014, pages 51-65, Coimbra, Portugal, July 2014. University of Coimbra.

[BNSB14b] Pierre Boutry, Julien Narboux, Pascal Schreck, and Gabriel Braun. Using small scale automation to improve both accessibility and readability of formal proofs in geometry. In Francisco Botana and Pedro Quaresma, editors, Proceedings of the 10th Int. Workshop on Automated Deduction in Geometry, volume TR 2014/01 of Proceedings of ADG 2014, pages 31-49, Coimbra, Portugal, July 2014. University of Coimbra.

[BR16] Francisco Botana and Tomas Recio. On the unavoidable uncertainty of truth in dynamic geometry proving. Mathematics in Computer Science, 10(1):5-25, 2016.

[BS60] Karol Borsuk and Wanda Szmielew. Foundations of geometry. North-Holland, 1960.

[BW17] Michael Beeson and Larry Wos. Finding proofs in tarskian geometry. Journal of Automated Reasoning, 58(1):181-207, 2017.

[CGZ94] Shang-Ching Chou, Xiao-Shan Gao, and Jing-Zhong Zhang. Machine Proofs in Geometry. World Scientific, Singapore, 1994.

[CM12] Cyril Cohen and Assia Mahboubi. Formal proofs in real algebraic geometry: from ordered fields to quantifier elimination. Logical Methods in Computer Science, 8(1:02):1-40, February 2012.

[CW13] Xiaoyu Chen and Dongming Wang. Formalization and Specification of Geometric Knowledge Objects. Mathematics in Computer Science, 7(4):439-454, 2013.

[DDS01] Christophe Dehlinger, Jean-François Dufourd, and Pascal Schreck. Higher-Order Intuitionistic Formalization and Proofs in Hilbert's Elementary Geometry. In Automated Deduction in Geometry, volume 2061 of Lecture Notes in Computer Science, pages 306-324. Springer, 2001. 
[Des25] René Descartes. La géométrie. Open Court, Chicago, 1925. first published as an appendix to the Discours de la Méthode (1637).

[EHD02] Euclid, T.L. Heath, and D. Densmore. Euclid's Elements: all thirteen books complete in one volume : the Thomas L. Heath translation. Green Lion Press, 2002.

[FT11] Laurent Fuchs and Laurent Théry. A Formalization of Grassmann-Cayley Algebra in COQ and Its Application to Theorem Proving in Projective Geometry, pages 5167. Springer, Berlin, Heidelberg, 2011.

[GNS11] Jean-David Genevaux, Julien Narboux, and Pascal Schreck. Formalization of Wu's simple method in Coq. In Jean-Pierre Jouannaud and Zhong Shao, editors, CPP 2011 First International Conference on Certified Programs and Proofs, volume 7086 of Lecture Notes in Computer Science, pages 71-86, Kenting, Taiwan, December 2011. Springer-Verlag.

[GPT11] Benjamin Grégoire, Loïc Pottier, and Laurent Théry. Proof Certificates for Algebra and their Application to Automatic Geometry Theorem Proving. In Postproceedings of Automated Deduction in Geometry (ADG 2008), number 6701 in Lecture Notes in Artificial Intelligence, 2011.

[Gro61] School Mathematics Study Group. Mathematics for high school: Geometry. Teacher's commentary. Yale University Press, New Haven, 1961.

[Gup65] Haragauri Narayan Gupta. Contributions to the axiomatic foundations of geometry. $\mathrm{PhD}$ thesis, University of California, Berkley, 1965.

[Hil60] David Hilbert. Foundations of Geometry (Grundlagen der Geometrie). Open Court, La Salle, Illinois, 1960. Second English edition, translated from the tenth German edition by Leo Unger. Original publication date, 1899.

[Jan06] Predrag Janičić. GCLC - A Tool for Constructive Euclidean Geometry and More than That. In Nobuki Takayama, Andres Iglesias, and Jaime Gutierrez, editors, Proceedings of International Congress of Mathematical Software (ICMS 2006), volume 4151 of Lecture Notes in Computer Science, pages 58-73. Springer-Verlag, 2006.

[JNQ12] Predrag Janičić, Julien Narboux, and Pedro Quaresma. The Area Method : a Recapitulation. Journal of Automated Reasoning, 48(4):489-532, 2012.

[Kle93a] Felix Klein. A comparative review of recent researches in geometry. Bull. New York Math. Soc., 2(10):215-249, 071893.

[Kle93b] Felix Klein. Vergleichende betrachtungen über neuere geometrische forschungen. Mathematische Annalen, 43(1):63-100, 1893.

[MF03] Laura I. Meikle and Jacques D. Fleuriot. Formalizing Hilbert's Grundlagen in Isabelle/Isar, pages 319-334. Springer Berlin Heidelberg, Berlin, Heidelberg, 2003.

[Moi90] E.E. Moise. Elementary Geometry from an Advanced Standpoint. Addison-Wesley, 1990.

[MP91] Richard S Millman and George D Parker. Geometry: a metric approach with models. Springer Science \& Business Media, 1991.

[MP15] Filip Marić and Danijela Petrović. Formalizing complex plane geometry. Annals of Mathematics and Artificial Intelligence, 74(3-4):271-308, 2015.

[MPPJ12] Filip Marić, Ivan Petrović, Danijela Petrović, and Predrag Janičić. Formalization and implementation of algebraic methods in geometry. In Pedro Quaresma and Ralph-Johan Back, editors, Proceedings First Workshop on CTP Components for Educational Software, Wrocław, Poland, 31th July 2011, volume 79 of Electronic Proceedings in Theoretical Computer Science, pages 63-81. Open Publishing Association, 2012. 
[MS16] P. Mathis and P. Schreck. Determining automatically compass and straightedge unconstructibility in triangles. In James H. Davenport and Fadoua Ghourabi, editors, 7th International Symposium on Symbolic Computation, volume 39 of EPiC Series in Computing, pages 130-142. James H. Davenport and Fadoua Ghourabi (EasyChair), Mar 2016. issn 2040-557X.

[Nar04] Julien Narboux. A Decision Procedure for Geometry in Coq. In Slind Konrad, Bunker Annett, and Gopalakrishnan Ganesh, editors, Proceedings of TPHOLs'2004, volume 3223 of Lecture Notes in Computer Science. SpringerVerlag, 2004.

[Nar07] Julien Narboux. Mechanical Theorem Proving in Tarski's geometry. In Francisco Botana Eugenio Roanes Lozano, editor, Post-proceedings of Automated Deduction in Geometry 2006, volume 4869 of LNCS, pages 139-156, Pontevedra, Spain, 2007. Francisco Botana, Springer.

[NB16] Julien Narboux and David Braun. Towards A Certified Version of the Encyclopedia of Triangle Centers. Mathematics in Computer Science, 10(1):17, April 2016.

[Pam98] Victor Pambuccian. Zur existenz gleichseitiger dreiecke in h-ebenen. Journal of Geometry, 63(1):147-153, 1998.

[RGA14] William Richter, Adam Grabowski, and Jesse Alama. Tarski geometry axioms. Formalized Mathematics, 22(2):167-176, 2014.

[SDNJ15] Sana Stojanović Durdević, Julien Narboux, and Predrag Janičić. Automated Generation of Machine Verifiable and Readable Proofs: A Case Study of Tarski's Geometry. Annals of Mathematics and Artificial Intelligence, page 25, 2015.

[Soz10] Matthieu Sozeau. A new look at generalized rewriting in type theory. Journal of Formalized Reasoning, 2(1):41-62, 2010.

[SST83] Wolfram Schwabhäuser, Wanda Szmielew, and Alfred Tarski. Metamathematische Methoden in der Geometrie. Springer-Verlag, Berlin, 1983.

[TG99] Alfred Tarski and Steven Givant. Tarski's system of geometry. The bulletin of Symbolic Logic, 5(2), June 1999.

[Wu94] Wen-Tsün Wu. Mechanical Theorem Proving in Geometries. Springer-Verlag, 1994.

[YCG08] Zheng Ye, Shang-Ching Chou, and Xiao-Shan Gao. An introduction to java geometry expert. In International Workshop on Automated Deduction in Geometry, volume 6301 of LNCS, pages 189-195. Springer, 2008. 


\section{A. Definitions of the Geometric Predicates}

\begin{tabular}{|c|c|c|}
\hline Coq & Notation & Definition \\
\hline Bet A B C & $A-B-C$ & \\
\hline Cong A B C D & $A B \equiv C D$ & \\
\hline Cong_3 A $B$ C $A^{\prime} \quad B^{\prime} \quad C^{\prime}$ & & $A B \equiv A^{\prime} B^{\prime} \wedge A C \equiv A^{\prime} C^{\prime} \wedge B C \equiv B^{\prime} C^{\prime}$ \\
\hline Col A B C & $\mathrm{Col} A B C$ & $A-B-C \vee B-A-C \vee A-C-B$ \\
\hline Out $\bigcirc \mathrm{A} B$ & & $O \neq A \wedge O \neq B \wedge(O-A-B \vee O-B-A)$ \\
\hline Midpoint M A B & $A_{+} M_{+} B$ & $A-M-B \wedge A M \equiv B M$ \\
\hline Per A B C & $\triangle A B C$ & $\exists C^{\prime}, C+B+C^{\prime} \wedge A C \equiv A C^{\prime}$ \\
\hline Perp_at P A B C D & $A B \underset{P}{\perp} C D$ & $\begin{array}{l}A \neq B \wedge C \neq D \wedge \operatorname{Col} P A B \wedge \operatorname{Col} P C D \wedge \\
(\forall U V, \operatorname{Col} U A B \Rightarrow \operatorname{Col} V C D \Rightarrow \triangle U P V)\end{array}$ \\
\hline Perp A B C D & $A B \perp C D$ & $\exists P, A B \underset{P}{\perp} C D$ \\
\hline Coplanar A B C D & Cp $A B C D$ & $\begin{array}{l}\exists X,(\mathrm{Col} A B X \wedge \mathrm{Col} C D X) \vee(\mathrm{Col} A C X \wedge \\
\mathrm{Col} B D X) \vee(\mathrm{Col} A D X \wedge \mathrm{Col} B C X))\end{array}$ \\
\hline Par_strict A B C D & $A B \|_{s} C D$ & $\begin{array}{l}A \neq B \wedge C \neq D \wedge \operatorname{Cp} A B C D \wedge \\
\neg \exists X, \operatorname{Col} X A B \wedge \operatorname{Col} X C D\end{array}$ \\
\hline Par A B C D & $A B \| X Y$ & $\begin{array}{l}A B \|_{s} C D \vee(A \neq B \wedge C \neq D \wedge \operatorname{Col} A C D \wedge \\
\operatorname{Col} B C D)\end{array}$ \\
\hline $\operatorname{Proj} P$ Q A B X Y & & $\begin{array}{l}A \neq B \wedge X \neq Y \wedge \neg A B \| X Y \wedge \operatorname{Col} A B Q \wedge \\
(P Q \| X Y \vee P=Q)\end{array}$ \\
\hline Pj $A$ B C $C$ & & $A B \| C D \vee C=D$ \\
\hline Opp $\bigcirc \mathrm{E} \mathrm{E}^{\prime} \mathrm{A} \quad \mathrm{B}$ & & Sum $O E E^{\prime} B A O$ \\
\hline Diff $O E E^{\prime} A B C$ & & $\exists B^{\prime}$, opp $O E E^{\prime} B B^{\prime} \wedge$ sum $O E E^{\prime} A B^{\prime} C$ \\
\hline Projp P Q A B & & $\begin{array}{l}A \neq B \wedge((\mathrm{Col} A B Q \wedge A B \perp P Q) \vee(\mathrm{Col} A B P \wedge \\
P=Q))\end{array}$ \\
\hline Length $O \mathrm{E} \mathrm{E}^{\prime} \mathrm{A} \mathrm{B} \mathrm{L}$ & & $O \neq E \wedge \mathrm{Col} O E L \wedge \operatorname{LeP} O E E^{\prime} O L \wedge O L \equiv A B$ \\
\hline Prodg $O \mathrm{E} \mathrm{E}^{\prime} \mathrm{A} \quad \mathrm{B} C$ & & $\begin{array}{l}\text { Prod } O E E^{\prime} A B C \vee\left(\neg \operatorname{Ar} 2 O E E^{\prime} A B B \wedge C=\right. \\
O)\end{array}$ \\
\hline
\end{tabular}

Table A.1. Definitions of the geometric predicates. 